\title{
Water-Borne Wood Preservation and End-of-Life Removal History and Projection
}

\author{
Stephen T. Smith (1) \\ Stephen Smith Consulting, Helena, MT, USA \\ Email: stephen@stephensmithconsulting.com
}

How to cite this paper: Smith, S.T. (2020) Water-Borne Wood Preservation and End-of-Life Removal History and Projection. Engineering, 12, 117-139. https://doi.org/10.4236/eng.2020.122011

Received: January 10, 2020

Accepted: February 25, 2020

Published: February 28, 2020

Copyright $\odot 2020$ by author(s) and Scientific Research Publishing Inc. This work is licensed under the Creative Commons Attribution International License (CC BY 4.0).

http://creativecommons.org/licenses/by/4.0/

\begin{abstract}
Use of water-borne wood preservatives began in approximately the 1950s. Residential and commercial uses rapidly developed for products such as decking, fences, and other outdoor structures. Nearly all such products were treated by preservatives using arsenic as a major ingredient. The most common preservative was chromated copper arsenate (CCA). A smaller volume used ammoniacal copper zinc arsenate (ACZA). Preservative label changes made in 2003 limited uses of these arsenical treatments to industrial or agricultural type uses, such as poles, piles, ties, bridges, and fencing. Use volumes of preservative-treated wood continued to grow after the label change, but the types of preservatives used changed greatly. The amounts of water-borne treated wood reaching end-of-life and being disposed also continued to grow, reflecting the increasing inventory of volume in service. However, the volume of arsenical-treated wood being disposed peaked in approximately 2008 and is now only approximately one-quarter of that volume. Most of the arsenical-treated wood now being disposed consists of large, easily identified and separated pieces, such as round poles, piles, and fence posts and timbers, which can be easily managed separated from other wood construction and demolition (C \& D) waste. Thus, managing C \& D waste to limit arsenic contamination of potential products, such as mulch, will be much more practical than some have feared.
\end{abstract}

\section{Keywords}

Water-Borne, Wood, Preservative, Arsenic, Copper, Disposal, CCA, C \& D, Landfill

\section{Introduction}

Nearly all preservative treated wood used in U. S. residential and commercial construction is a water-borne variety of preservative. This contrasts with oil-borne 
preservatives such as pentachlorophenol, creosote, and copper naphthenate that are used mainly for industrial products such as railroad ties, utility poles, piling, and other marine and highway construction. While also used for industrial products, water-borne treatment of wood for use as decks and fencing in residential and commercial applications started to become common during the 1950s. The residential and commercial uses of preservative treated lumber rapidly became very popular, reaching annual production of over 400 million cubic feet by 1987 [1].

Water-borne treatments from inception to 2003 primarily arsenical treatments, meaning that the preservative included arsenic as an active ingredient. Chromated copper arsenate (CCA) was most common and ammoniacal copper zinc arsenate (ACZA) was used less, but could be used for Douglas fir lumber, which does not treat well with CCA.

Public and regulatory concerns regarding human contact with arsenic from wood products, and arsenic in the waste streams, were developed in the 1990s and 2000s. In 2003, the wood preservative manufacturers voluntarily changed the label approved end uses of CCA and ACZA so that residential and commercial uses would not continue and only "heavy duty" industrial and agricultural products would be treated with these preservatives. Thus, annual treatment volume by arsenical preservatives (CCA and ACZA) dropped from nearly 600 million cubic feet to approximately 100 million cubic feet.

Alternative non-arsenical water-borne preservatives were developed and almost immediately became common. Copper azole (CA) and alkaline copper quat (ACQ) preservatives were standardized in several evolving types by the American Wood Protection Association (AWPA) in the late 1990s and early 2000s. Dissolved copper provided most of the decay protection in these systems. Most lumber treated for residential decks and fences used these preservatives.

Preservatives using fine particles of copper, called micronized copper, were approved by the International Codes Council Engineering Service (ICC ES) and began to be commonly used after 2010. The main preservatives of this type were micronized copper azole (MCA), standardized by AWPA in 2016, and micronized copper quat (MCQ). MCA now leads the residential and commercial outdoor lumber market. Other non-arsenic copper-based preservatives currently in the market include Copper HDO (CX-A), Alkaline Copper Betaine (KDS and KDS-B), and water-borne Copper Naphthenate (CuN-W).

Newer preservative systems have recently been marketed for above ground exposures that do not include any metals, but rely on organic pesticides only. Two of these are Ecolife (EL2), using active ingredients 4,5-dichloro-2-N-Octyl4-Isothiazolin-3-One (DCOI) and Imidacloprid, and Propiconazole-Tebuconazole-Imidacloprid (PTI).

The focus of this report is on dimensional residential and commercial preservative treated lumber for exterior applications. Thus, borate preservative use is not addressed in the report.

While the change of preservatives away from those containing arsenic has 
largely addressed concerns about exposure to the treated product, concerns remain regarding potential environmental impacts associated with disposal of arsenical wood products treated prior to 2004 and for arsenical-approved products since then. In particular, the U. S. Environmental Protection Agency (EPA) requested information about disposal as part of its 2008 process to reauthorize uses of the preservatives. CCA and ACZA use was re-authorized by the EPA in 2008.

In 2005, the Wood Preservative Science Council (WPSC) sponsored a study [2] on the impact of the voluntary label change that eliminated most residential and commercial uses of CCA-treated wood. That study provided the amounts of CCA preservative used in the U.S. before and the year after the label change and estimated the amounts of wood treated by CCA for the same times. The volume of wood treated with CCA dropped from nearly 600 million cubic feet in 2000 down to an estimated 109 million cubic feet in 2004. The volume after 2003 was approximately $18 \%$ of the volume before that year. That study also estimated disposal rates of CCA treated wood in the U.S. Disposal volumes were estimated using estimated average service lives and standard deviations for the treated products with historic reports of wood treatment volumes. After 2004, annual volume of CCA treatment was assumed to be 100 million cubic feet. This study was provided to the EPA, but was not published.

In 2016, the Arsenical Treated Wood Task Force (ATWTF) sponsored a study [3] on the amount of CCA preservative used, and the products for which it was used as a follow up to the 2005 study. This study used both data on total CCA preservative sold in the U. S. and a survey of CCA wood preserving companies. The total CCA preservative used by U.S. treating companies in 2014 was reported to the author by the client as just under $50,000,000$ pounds. That amount (50 million pounds) was accepted as the amount used in 2015. CCA use from this time forward was expected to be relatively stable, since the residential market was no longer part of the CCA-treated wood market. A survey of treaters was used to determine the typical product fractions of the total market treated with CCA. Use of CCA preservatives was estimated for the reported products based on required preservative retentions, typical treatable fractions for the wood products, and typical assumed overtreatment to assure retentions are met. Volumes were projected base on the assumed total CCA preservative use of 50 million pounds. This resulted in a projected total CCA-treated wood volume of 78.7 million cubic feet. Again, this study was provided to the EPA, but was not published.

Reflecting on-going concern about arsenic and other metals in the waste disposal stream, Jones et al. [4] recently evaluated potential contamination of construction and demolition (C \& D) waste utilized for mulch. The authors used published data, industry standards, and assumptions to estimate the amounts of water-borne preservative treated wood entering the waste stream annually. They concluded, in part, that levels of arsenic in C \& D waste due to treated wood would "greatly limit the recycling potential for C \& D wood." However, since 
their work was based on published data, it did not include references such as the two listed above. As a result, the amount of arsenic in treated wood waste was over-estimated and the products in the waste were incorrectly characterized as mostly lumber rather than larger dimension timber and round wood.

Published articles, such as the one described above, that mischaracterize treated wood waste and generally contribute to misinformation about treated wood, highlights the need to make more accurate information about treated wood publicly available. Recognizing this need, the Treated Wood Council (TWC) has commissioned this survey and report.

The evaluation and survey covered by this report seek to provide accurate information about historic, current, and near future projected treatment, use, and disposition of wood products treated with water-borne wood preservatives. This goal includes documenting the transition from arsenic based preservatives, primarily chromated copper arsenate (CCA), to water-borne preservatives not containing arsenic.

\section{Methods}

Information from previously published documents and some public, but unpublished documents, regarding wood preservatives combined with new survey data about the amounts of water-borne wood preservatives used in the U.S. in recent years has been used, along with wood preservation standards and professional judgement, to estimate volumes of water-borne preservative products annual production and end-of-life removal from service from 1950 to 2050. Annual production is subdivided into production of various products and preservatives used for each product. Products produced are assumed to be put into service in the year of production. Typical service life for each product (average service life and standard deviation) is estimated for each product (The author used several life cycle assessments, industry data, and professional judgement to make these estimates). At end-of-life, products are assumed to be disposed.

Estimates of production of water-borne preserved wood products over past decades were developed using several reports by the wood preserving industry. [1] [5] [6]. These reports were previously used by this author to complete a report on CCA treatment [2] and to prepare a chapter regarding the economics of treated wood [7].

Wood preservation standards, such as minimum retentions and preservative solutions composition, have been obtained from the AWPA Book of Standards [8].

Current preserved wood production by various water-borne preservatives is estimated by using the results of a survey conducted jointly by this author and the TWC of preservative suppliers in the U.S. The survey obtained U.S. sales amounts of arsenic based, non-arsenic copper based, and non-metal wood preservatives sold in 2016 and 2018. Survey results of preservative volumes do not include all preservative suppliers. It is estimated that $95 \%$ of arsenical and $80 \%$ 
of non-arsenic copper preservatives are covered. However, only a small portion of the non-metal preservative volume was reported. Volumes of preserved wood products were estimated using previously estimated wood product type proportions, minimum retentions of preservatives for each product, proportions of non-metal preserved products, and author professional judgement, which included estimates in years 2002 through 2015 to smooth total annual production values.

\section{Results}

\subsection{Production}

Statistical reports of wood preservation annual amounts generally categorized preservation into three categories; creosote, oil-borne, and water-borne preservatives. Only the water-borne preservatives are applicable to this report. Prior to 2004, water-borne preservative was nearly all arsenic-based with approximately $96 \%$ being chromated copper arsenate (CCA) and $4 \%$ being ammoniacal copper zinc arsenate (ACZA). In 2005, an estimate of annual production of CCA treatment from 1950 through 2004 was prepared using these statistical reports along with a 2005 survey of CCA preservative sales from 1996 through 2004. Annual production from 2005 and forward was assumed constant.

In this report, annual production of water-borne preservative treated wood products is the sum of arsenical, non-arsenical copper, and non-metal preservatives and is made based on the survey of 2016 and 2018 preservative sales that is associated with this report. The survey results were refined using AWPA retentions and author professional judgement including product mix estimates. Following 2018, annual production is assumed to increase at $1 \%$ per year for each category of preservatives. The resulting annual production of water-borne preservatives from 1950 to 2050 is shown in Table 1.

As noted, a survey of water-borne preservative suppliers was conducted to support this report. A copy of the survey form may be obtained from the author. Suppliers reported sales directly to this author. They remain confidential. A summary of the survey results is included as Table 2. Note that professional judgement was applied to make assumptions regarding the product and preservative mix associated with each preservative category reported in order to calculate estimated product volume and associated arsenic and copper contained in those products.

The AWPA standards require minimum retentions of preservatives for various products based on the service conditions in which they will be used. Use Categories define these exposure conditions and the associated degrees of decay and insect attack hazard. Use Category numbers increase with increasing hazard. UC 1 and UC2 apply to interior applications and UC3A to coated exterior wood with little hazard. UC3B applies to exterior, uncoated, above-ground applications, such as decks, that get and remain wet and are subject to decay and insect attack. UC4 covers situations where the products are in contact with ground 
Table 1. Production and disposal by year.

\begin{tabular}{|c|c|c|c|c|c|c|c|c|c|}
\hline \multicolumn{10}{|c|}{ Production of Water-Borne Preserved Wood by Year } \\
\hline \multicolumn{4}{|c|}{ Copied from Smith, 2005, Table 1} & \multicolumn{4}{|c|}{ Annual Treatment Volume } & \multicolumn{2}{|c|}{ Disposal Volume } \\
\hline $\begin{array}{l}\text { Treatment } \\
\text { Year }\end{array}$ & $\begin{array}{l}\text { Reference/ } \\
\text { Method }\end{array}$ & $\begin{array}{c}\text { Waterborne } \\
\text { Treatment } \\
(1000 \mathrm{cf})\end{array}$ & $\begin{array}{c}\text { CCA } \\
\text { Treatment } \\
(1000 \mathrm{cf})\end{array}$ & $\begin{array}{l}\text { Arsenical } \\
\text { Treatments }\end{array}$ & $\begin{array}{c}\text { Non-As } \\
\text { Copper } \\
\text { Treatments }\end{array}$ & $\begin{array}{c}\text { Other } \\
\text { non-metal } \\
\text { treatments }\end{array}$ & $\begin{array}{c}\text { All } \\
\text { Treatments }\end{array}$ & $\begin{array}{c}\text { Arsenical } \\
\text { TW } \\
\text { Disposal }\end{array}$ & $\begin{array}{c}\text { All TW } \\
\text { Disposal }\end{array}$ \\
\hline \multicolumn{5}{|c|}{$(1000 \mathrm{cf})$} & \multicolumn{2}{|c|}{ (Volume in $1000 \mathrm{cf}$ ) } & \multicolumn{3}{|c|}{$(1000 \mathrm{cf})$} \\
\hline 1950 & Assumed value & 0 & 0 & 0 & 0 & 0 & 0 & 0 & 0 \\
\hline 1951 & & 425 & 408 & 425 & 0 & 0 & 425 & 9 & 9 \\
\hline 1952 & & 851 & 817 & 851 & 0 & 0 & 851 & 19 & 19 \\
\hline 1953 & & 1276 & 1225 & 1276 & 0 & 0 & 1276 & 28 & 28 \\
\hline 1954 & & 1702 & 1634 & 1702 & 0 & 0 & 1702 & 40 & 40 \\
\hline 1955 & & 2127 & 2042 & 2127 & 0 & 0 & 2127 & 61 & 61 \\
\hline 1956 & & 2553 & 2451 & 2553 & 0 & 0 & 2553 & 97 & 97 \\
\hline 1957 & & 2978 & 2859 & 2978 & 0 & 0 & 2978 & 156 & 156 \\
\hline 1958 & Straight line & 3404 & 3267 & 3404 & 0 & 0 & 3404 & 242 & 242 \\
\hline 1959 & $\begin{array}{l}\text { proportion } \\
\text { from }\end{array}$ & 3829 & 3676 & 3829 & 0 & 0 & 3829 & 356 & 356 \\
\hline 1960 & 1950 to 1968 & 4254 & 4084 & 4254 & 0 & 0 & 4254 & 500 & 500 \\
\hline 1961 & & 4680 & 4493 & 4680 & 0 & 0 & 4680 & 672 & 672 \\
\hline 1962 & & 5105 & 4901 & 5105 & 0 & 0 & 5105 & 871 & 871 \\
\hline 1963 & & 5531 & 5310 & 5531 & 0 & 0 & 5531 & 1096 & 1096 \\
\hline 1964 & & 5956 & 5718 & 5956 & 0 & 0 & 5956 & 1345 & 1345 \\
\hline 1965 & & 6382 & 6126 & 6382 & 0 & 0 & 6382 & 1614 & 1614 \\
\hline 1966 & & 807 & 6535 & 6807 & 0 & 0 & 6807 & 1902 & 1902 \\
\hline 1967 & & 7233 & 6943 & 7233 & 0 & 0 & 7233 & 2207 & 2207 \\
\hline 1968 & & 7658 & 7352 & 7658 & 0 & 0 & 7658 & 2524 & 2524 \\
\hline 1969 & & 10,514 & 10,093 & 10,514 & 0 & 0 & 10,514 & 2907 & 2907 \\
\hline 1970 & & 15,135 & 14,530 & 15,135 & 0 & 0 & 15,135 & 3338 & 3338 \\
\hline 1971 & & 20,251 & 19,441 & 20,251 & 0 & 0 & 20,251 & 3789 & 3789 \\
\hline 1972 & Maloney \& & 25,633 & 24,608 & 25,633 & 0 & 0 & 25,633 & 4267 & 4267 \\
\hline 1973 & $\begin{array}{l}\text { Pagliai, } 1978, \\
\text { Table } 18\end{array}$ & 29,414 & 28,237 & 29,414 & 0 & 0 & 29,414 & 4772 & 4772 \\
\hline 1974 & & 41,072 & 39,429 & 41,072 & 0 & 0 & 41,072 & 5586 & 5586 \\
\hline 1975 & & 29,851 & 28,657 & 29,851 & 0 & 0 & 29,851 & 6105 & 6105 \\
\hline 1976 & & 44,781 & 42,990 & 44,781 & 0 & 0 & 44,781 & 7469 & 7469 \\
\hline 1977 & & 42,661 & 40,955 & 42,661 & 0 & 0 & 42,661 & 8772 & 8772 \\
\hline 1978 & & & 62,319 & 64,916 & 0 & 0 & 64,916 & 10,922 & 10,922 \\
\hline 1979 & Ferry 1982 & Reported as & 95,690 & 99,677 & 0 & 0 & 99,677 & 13,585 & 13,585 \\
\hline 1980 & гену, 1902 & CCA Trmt. & 111,012 & 115,638 & 0 & 0 & 115,638 & 16,044 & 16,044 \\
\hline 1981 & & & 131,292 & 136,763 & 0 & 0 & 136,763 & 18,896 & 18,896 \\
\hline
\end{tabular}


S. T. Smith

Continued

\begin{tabular}{|c|c|c|c|c|c|c|c|c|c|}
\hline 1982 & Interpolated & & 185,263 & 192,982 & 0 & 0 & 192,982 & 23,085 & 23,085 \\
\hline 1983 & Micklewright, & 249,202 & 239,234 & 249,202 & 0 & 0 & 249,202 & 28,234 & 28,234 \\
\hline 1984 & 1986 & 301,697 & 289,629 & 301,697 & 0 & 0 & 301,697 & 34,534 & 34,534 \\
\hline 1985 & & 328,677 & 315,530 & 328,677 & 0 & 0 & 328,677 & 41,773 & 41,773 \\
\hline 1986 & & 375,458 & 360,440 & 375,458 & 0 & 0 & 375,458 & 51,445 & 51,445 \\
\hline 1987 & AWPI, 1997 & 418,984 & 402,225 & 418,984 & 0 & 0 & 418,984 & 63,613 & 63,613 \\
\hline 1988 & & 450,565 & 432,542 & 450,565 & 0 & 0 & 450,565 & 78,393 & 78,393 \\
\hline 1989 & & 406,941 & 390,663 & 406,941 & 0 & 0 & 406,941 & 94,324 & 94,324 \\
\hline 1990 & & 437,675 & 420,168 & 437,675 & 0 & 0 & 437,675 & 114,509 & 114,509 \\
\hline 1991 & 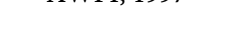 & 424,370 & 407,395 & 424,370 & 0 & 0 & 424,370 & 136,067 & 136,067 \\
\hline 1992 & Interpolated & 447,437 & 429,540 & 447,437 & 0 & 0 & 447,437 & 160,079 & 160,079 \\
\hline 1993 & & 470,504 & 451,684 & 470,504 & 0 & 0 & 470,504 & 184,636 & 184,636 \\
\hline 1994 & & 496,883 & 477,008 & 496,883 & 0 & 0 & 496,883 & 208,884 & 208,884 \\
\hline 1995 & क्ष & 450,596 & 432,572 & 450,596 & 0 & 0 & 450,596 & 230,578 & 230,578 \\
\hline 1996 & & 467,855 & 449,141 & 467,855 & 0 & 0 & 467,855 & 252,573 & 252,573 \\
\hline 1997 & $\begin{array}{c}\text { Micklewright, } \\
1998\end{array}$ & 581,382 & 558,127 & 581,382 & 0 & 0 & 581,382 & 275,709 & 275,709 \\
\hline 1998 & & & 540,204 & 562,713 & 0 & 0 & 562,713 & 294,857 & 294,857 \\
\hline 1999 & & & 588,427 & 612,944 & 0 & 0 & 612,944 & 314,037 & 314,037 \\
\hline 2000 & $\begin{array}{l}\text { Calculated } \\
\text { from } 2005\end{array}$ & & 599,525 & 624,505 & 0 & 0 & 624,505 & 331,083 & 331,083 \\
\hline 2001 & $\begin{array}{l}\text { survey of } \\
\text { chemical }\end{array}$ & & 571,134 & 594,931 & 0 & 0 & 594,931 & 346,856 & 346,856 \\
\hline 2002 & $\begin{array}{l}\text { suppliers } \\
\text { (Table 3) }\end{array}$ & & 541,873 & 564,451 & 25,000 & 0 & 589,451 & 362,882 & 363,434 \\
\hline 2003 & & & 505,603 & 526,670 & 50,000 & 0 & 576,670 & 379,233 & 380,336 \\
\hline 2004 & & & 109,052 & 113,596 & 380,000 & 10,000 & 503,596 & 385,361 & 396,108 \\
\hline 2005 & & & & 85,000 & 427,500 & 10,000 & 522,500 & 401,985 & 413,395 \\
\hline 2006 & & & & 85,000 & 427,500 & 10,000 & 522,500 & 416,783 & 429,026 \\
\hline 2007 & $\begin{array}{l}\text { Vlosky est } 608 \mathrm{~m} \\
\text { cf water-borne }\end{array}$ & & & 85,000 & 475,000 & 10,000 & 570,000 & 426,453 & 443,728 \\
\hline 2008 & & & & 85,000 & 475,000 & 20,000 & 580,000 & 426,162 & 454,971 \\
\hline 2009 & & & & 85,000 & 475,000 & 20,000 & 580,000 & 414,072 & 463,347 \\
\hline 2010 & & & & 85,000 & 475,000 & 20,000 & 580,000 & 392,167 & 469,737 \\
\hline 2011 & & & & 85,000 & 475,000 & 25,000 & 585,000 & 363,831 & 475,216 \\
\hline 2012 & & & & 85,000 & 475,000 & 25,000 & 585,000 & 332,006 & 480,227 \\
\hline 2013 & & & & 85,000 & 475,000 & 25,000 & 585,000 & 298,752 & 485,067 \\
\hline 2014 & & & & 85,000 & 475,000 & 25,000 & 585,000 & 265,480 & 489,701 \\
\hline 2015 & 2016 Survey Value & & 78,665 & 81,943 & 475,000 & 25,000 & 581,943 & 233,239 & 494,033 \\
\hline
\end{tabular}




\section{Continued}

\begin{tabular}{|c|c|c|c|c|c|c|}
\hline 2016 & 70,608 & 473,851 & 23,541 & 568,000 & 202,851 & 497,904 \\
\hline 2017 & 70,355 & 502,847 & 32,679 & 605,881 & 175,016 & 502,718 \\
\hline 2018 & 70,102 & 531,843 & 41,816 & 643,761 & 150,117 & 507,326 \\
\hline 2019 & 70,803 & 537,161 & 42,235 & 650,199 & 128,358 & 510,963 \\
\hline 2020 & 71,511 & 542,533 & 42,657 & 656,701 & 109,746 & 514,383 \\
\hline 2021 & 72,226 & 547,958 & 43,084 & 663,268 & 94,139 & 518,057 \\
\hline 2022 & 72,948 & 553,438 & 43,514 & 669,900 & 81,296 & 522,570 \\
\hline 2023 & 73,677 & 558,972 & 43,950 & 676,599 & 70,912 & 528,155 \\
\hline 2024 & 74,414 & 564,562 & 44,389 & 683,365 & 62,659 & 534,685 \\
\hline 2025 & 75,158 & 570,208 & 44,833 & 690,199 & 56,211 & 541,920 \\
\hline 2026 & 75,910 & 575,910 & 45,281 & 697,101 & 51,264 & 549,653 \\
\hline 2027 & 76,669 & 581,669 & 45,734 & 704,072 & 47,544 & 557,743 \\
\hline 2028 & 77,436 & 587,486 & 46,191 & 711,113 & 44,818 & 566,088 \\
\hline 2029 & 78,210 & 593,360 & 46,653 & 718,224 & 42,890 & 574,607 \\
\hline 2030 & 78,992 & 599,294 & 47,120 & 725,406 & 41,601 & 583,226 \\
\hline 2031 & 79,782 & 605,287 & 47,591 & 732,660 & 40,829 & 591,883 \\
\hline 2032 & 80,580 & 611,340 & 48,067 & 739,987 & 40,479 & 600,530 \\
\hline 2033 & 81,386 & 617,453 & 48,548 & 747,386 & 40,478 & 609,133 \\
\hline 2034 & 82,200 & 623,628 & 49,033 & 754,860 & 40,774 & 617,672 \\
\hline 2035 & 83,022 & 629,864 & 49,523 & 762,409 & 41,321 & 626,137 \\
\hline 2036 & 83,852 & 636,163 & 50,019 & 770,033 & 42,086 & 634,527 \\
\hline 2037 & 84,690 & 642,524 & 50,519 & 777,733 & 43,034 & 642,847 \\
\hline 2038 & 85,537 & 648,949 & 51,024 & 785,511 & 44,139 & 651,104 \\
\hline 2039 & 86,393 & 655,439 & 51,534 & 793,366 & 45,371 & 659,309 \\
\hline 2040 & 87,256 & 661,993 & 52,050 & 801,299 & 46,707 & 667,473 \\
\hline 2041 & 88,129 & 668,613 & 52,570 & 809,312 & 48,121 & 675,606 \\
\hline 2042 & 89,010 & 675,299 & 53,096 & 817,406 & 49,593 & 683,719 \\
\hline 2043 & 89,900 & 682,052 & 53,627 & 825,580 & 51,103 & 691,821 \\
\hline 2044 & 90,799 & 688,873 & 54,163 & 833,835 & 52,634 & 699,921 \\
\hline 2045 & 91,707 & 695,762 & 54,705 & 842,174 & 54,170 & 708,024 \\
\hline 2046 & 92,625 & 702,719 & 55,252 & 850,596 & 55,699 & 716,136 \\
\hline 2047 & 93,551 & 709,747 & 55,804 & 859,101 & 57,209 & 724,260 \\
\hline 2048 & 94,486 & 716,844 & 56,362 & 867,692 & 58,690 & 732,401 \\
\hline 2049 & 95,431 & 724,012 & 56,926 & 876,369 & 60,134 & 740,561 \\
\hline 2050 & 96,385 & 731,253 & 57,495 & 885,133 & 61,534 & 748,744 \\
\hline
\end{tabular}


Table 2. Preservative production survey results.

\begin{tabular}{|c|c|c|c|c|}
\hline \multicolumn{5}{|c|}{2016} \\
\hline Preservative Category & Preservative Amt (lb) & Treated Wood Amt (cf) & Arsenic Amt (lb) & Copper Amt (lb) \\
\hline Basis: & Survey & \multicolumn{3}{|c|}{ Estimate \& Calculation } \\
\hline Arsenical & $49,670,133$ & $70,608,158$ & $10,897,195$ & $7,839,509$ \\
\hline Non-Arsenical Copper & $45,806,851$ & $473,851,043$ & 0 & $40,962,980$ \\
\hline Non-metal & 380,000 & $23,541,114$ & 0 & 0 \\
\hline Totals & $95,856,985$ & $568,000,314$ & $10,897,195$ & $48,802,489$ \\
\hline \multicolumn{5}{|c|}{2018} \\
\hline Preservative Category & Preservative Amt (lb) & Treated Wood Amt (cf) & Arsenic Amt (lb) & Copper Amt (lb) \\
\hline Basis: & Survey & \multicolumn{3}{|c|}{ Estimate \& Calculation } \\
\hline Arsenical & $49,313,732$ & $70,101,519$ & $10,819,004$ & $7,783,258$ \\
\hline Non-Arsenical Copper & $51,032,076$ & $531,842,974$ & 0 & $46,307,997$ \\
\hline Non-metal & 675,000 & $41,816,453$ & 0 & 0 \\
\hline Totals & $101,020,808$ & $643,760,945$ & $10,819,004$ & $54,091,254$ \\
\hline
\end{tabular}

and, therefore, are likely to remain wet for extended periods and also be in contact with soil bacteria and insects. UC 5 covers marine (salt water) exposures, which have the highest decay and insect attack potential. These Use Categories, conditions, applications, product commodities, and required minimum retentions for each preservative are summarized in Table 3. Since products used in UC1, 2, and 3A generally do not require preservative treatment, they are not applicable to this study.

Table 4 uses the same format as Table 3 to list the author's estimates of the fraction of each product currently treated by each preservative. The fractions for each preservative equal $100 \%$. For example, $52 \%$ of CCA is used to treat poles, but none is used to treat deck lumber (UC3B). Since only a small portion of non-metal preservative use was reported in the survey, it was necessary to estimate use by alternate means. A paper presented to the AWPA 2016 Annual Meeting [9] provided estimates of fractions of all treatment by various water-borne preservatives. Treatment market share estimates for 2014-2016 developed in this study are shown in Table 5. Since the amounts of non-metal preservatives were mostly not reported, preservative use was assumed so that the volumes of treated wood would approximately match the proportions estimated in this paper. Values from Table 2 and Table 4 are used to calculate estimated fractions for each commodity-preservative item as a percent of all treated wood based on the current product mix in Table 6 .

Also included as one column of Table 4 is treatable fraction. Wood preservatives do not penetrate and remain constant through the whole wood product. Typically, the interior of a product will contain less or even no preservative. Generally, heartwood does not accept any preservative. Thus, each product will have treatable and untreatable fractions. For example, a sawn 2 -inch by 6 -inch 
Table 3. Retentions by use category.






\begin{tabular}{|c|c|c|c|c|c|c|c|c|c|c|c|c|c|c|c|c|}
\hline UC4C & $\begin{array}{l}\text { Ground } \\
\text { contact or } \\
\text { fresh water } \\
\text { Critical } \\
\text { structural } \\
\text { components }\end{array}$ & $\begin{array}{l}\text { Exposed to } \\
\text { all weather } \\
\text { cycles, severe } \\
\text { environments } \\
\text { extreme } \\
\text { decay } \\
\text { potential }\end{array}$ & $\begin{array}{c}\text { Decay fungi } \\
\text { and insects } \\
\text { with extreme } \\
\text { potential for } \\
\text { biodeterioration }\end{array}$ & $\begin{array}{c}\text { Land \& } \\
\text { Freshwater } \\
\text { piling, } \\
\text { foundation piling, } \\
\text { crossties \& } \\
\text { utility poles } \\
\text { (severe } \\
\text { decay areas) }\end{array}$ & $\begin{array}{c}\text { Sawn } \\
\text { Product } \\
\text { Round } \\
\text { piling } \\
\text { Posts }\end{array}$ & 0.80 & 0.60 & NL & 0.8 & NL & 0.41 & 0.41 & NL & NL & NL & NL \\
\hline $\begin{array}{c}\text { UC } \\
4 \mathrm{~A}, \mathrm{~B} \\
\& \mathrm{C}\end{array}$ & $\begin{array}{l}\text { Ground } \\
\text { Contact }\end{array}$ & $\begin{array}{l}\text { Decay fungi } \\
\text { and insects } \\
\text { \& high value } \\
\text { products }\end{array}$ & $\begin{array}{l}\text { Decay fungi } \\
\text { and insects \& } \\
\text { high value } \\
\text { products }\end{array}$ & All & $\begin{array}{c}\text { Poles } \\
\text { Crossties }\end{array}$ & $\begin{array}{l}0.60 \\
\mathrm{NL}\end{array}$ & 0.60 & NL & 0.6 & NL & 0.31 & NL & $\mathrm{NL}$ & NL & NL & NL \\
\hline UC5A & $\begin{array}{l}\text { Salt or } \\
\text { brackish } \\
\text { water and } \\
\text { adjacent } \\
\text { mud zone } \\
\text { Northern } \\
\text { waters }\end{array}$ & $\begin{array}{l}\text { Continuous } \\
\text { marine } \\
\text { exposure } \\
\text { (salt water) }\end{array}$ & $\begin{array}{l}\text { Salt water } \\
\text { organisms }\end{array}$ & $\begin{array}{l}\text { Piling, } \\
\text { bulkheads, } \\
\text { bracing }\end{array}$ & $\begin{array}{c}\text { Sawn } \\
\text { Product } \\
\text { Piles }\end{array}$ & 1.5 & 1.5 & NL & NL & NL & NL & NL & NL & NL & NL & NL \\
\hline UC5B & $\begin{array}{c}\text { Salt or } \\
\text { brackish } \\
\text { water and } \\
\text { adjacent } \\
\text { mud zone } \\
\text { NJ to GA, } \\
\text { south of } \\
\text { SanFran }\end{array}$ & $\begin{array}{l}\text { Continuous } \\
\text { marine } \\
\text { exposure } \\
\text { (salt water) }\end{array}$ & $\begin{array}{c}\text { Salt water } \\
\text { organisms } \\
\text { Including } \\
\text { creosote } \\
\text { tolerant } \\
\text { Limnoria } \\
\text { tripunctata }\end{array}$ & $\begin{array}{l}\text { Piling, } \\
\text { bulkheads, } \\
\text { bracing }\end{array}$ & $\begin{array}{c}\text { Sawn } \\
\text { Product } \\
\text { Piles }\end{array}$ & 2.5 & 2.5 & NL & NL & NL & NL & NL & $\mathrm{NL}$ & $\mathrm{NL}$ & NL & NL \\
\hline UC5C & $\begin{array}{c}\text { Salt or } \\
\text { brackish } \\
\text { water and } \\
\text { adjacent } \\
\text { mud zone } \\
\text { South of GA, } \\
\text { Gulf Coast, } \\
\text { Hawaii, and } \\
\text { Puerto Rico }\end{array}$ & $\begin{array}{l}\text { Continuous } \\
\text { marine } \\
\text { exposure } \\
\text { (salt water) }\end{array}$ & $\begin{array}{c}\text { Salt water } \\
\text { organisms } \\
\text { Including } \\
\text { Martesia, } \\
\text { Sphaeroma }\end{array}$ & $\begin{array}{l}\text { Piling, } \\
\text { bulkheads, } \\
\text { bracing }\end{array}$ & $\begin{array}{l}\text { Sawn } \\
\text { Product } \\
\text { Piles }\end{array}$ & 2.5 & 2.5 & NL & NL & NL & NL & NL & NL & NL & NL & NL \\
\hline $\begin{array}{l}\text { UC 5A, } \\
B, \& C\end{array}$ & $\begin{array}{c}\text { Marine } \\
\text { Exposure }\end{array}$ & $\begin{array}{l}\text { Continuous } \\
\text { marine } \\
\text { exposure } \\
\text { (salt water) }\end{array}$ & $\begin{array}{l}\text { Salt water } \\
\text { organisms }\end{array}$ & All & Plywood & 2.5 & 2.5 & NL & NL & NL & NL & NL & NL & NL & NL & NL \\
\hline
\end{tabular}

Posts, Modified, Farm Use retentions same as for UC4C posts, except ACQ-D added at 0.60 pcf. Round buidling poles and posts, UC4B retentions same as sawn UC4C.

nominal piece of lumber will have more treatable wood than a 6-inch by 8 -inch timber. In addition to retention, AWPA states minimum assay zones in which retention is measured and penetration zones, which are deeper than the assay zones where preservative must penetrate but which need not reach the full retention. This information is used to calculate typical treatable fractions in Table 7. Those fractions are then used in Table 4.

In the lower rows of Table 4, retentions, treatable fraction, and overtreatment (assumed to be $10 \%$ as discussed below) are used to calculate average retentions applicable to the product mix of each preservative and the amounts of arsenic and copper contained in the mix of products. Note that in the wood preservation process, treaters generally inject a bit more preservative (over treatment) than the minimum to account for wood variability so that when pieces are assayed 
Table 4. Preservative uses by commodity.

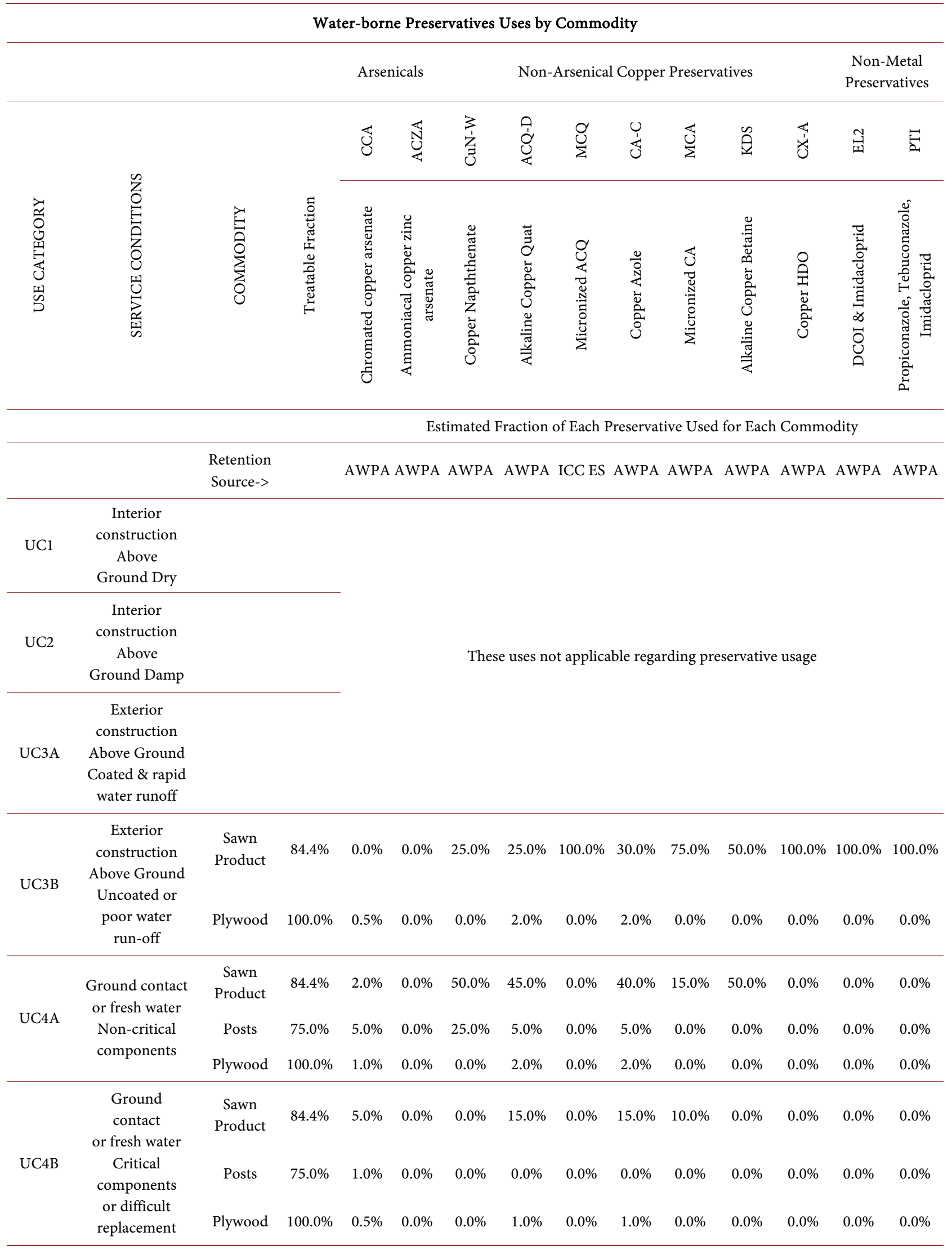




\section{Continued}



$10 \%$ Assumed actual treatment retention above minimum.

(tested), each is more likely to meet or exceed the minimum. In the 2016 study [3], overtreatment of $10 \%$ was found to fit reported data well and is used in this report. Since some retentions are specified for metal oxide fraction rather than pure metal fraction, conversions from oxide to pure metal fraction are made for the applicable preservatives in Table 8. 
Table 5. Solo-gabriel estimates of proportions of product by preservative.

\begin{tabular}{|c|c|c|c|c|c|c|c|}
\hline \multicolumn{8}{|c|}{ Proportions of Water-Borne Treatment Average 2014-2016 per Solo-Gabriele et al. 2016.} \\
\hline $\mathrm{CCA}$ & ACZA & ACQ & MCQ & $\mathrm{CA}$ & MCA & PTI & EL2 \\
\hline $1.0 \%$ & $0.0 \%$ & $8.5 \%$ & $0.0 \%$ & $10.9 \%$ & $66.7 \%$ & $2.4 \%$ & $10.5 \%$ \\
\hline \multicolumn{2}{|c|}{ Arsenical } & \multicolumn{4}{|c|}{ Copper non-arsenical } & \multicolumn{2}{|c|}{ Non-metal } \\
\hline $100.0 \%$ & $0 \%$ & $9.9 \%$ & $0.0 \%$ & $12.6 \%$ & $77.5 \%$ & $18.4 \%$ & $81.6 \%$ \\
\hline
\end{tabular}

Table 6. Product fractions by preservative and commodity.

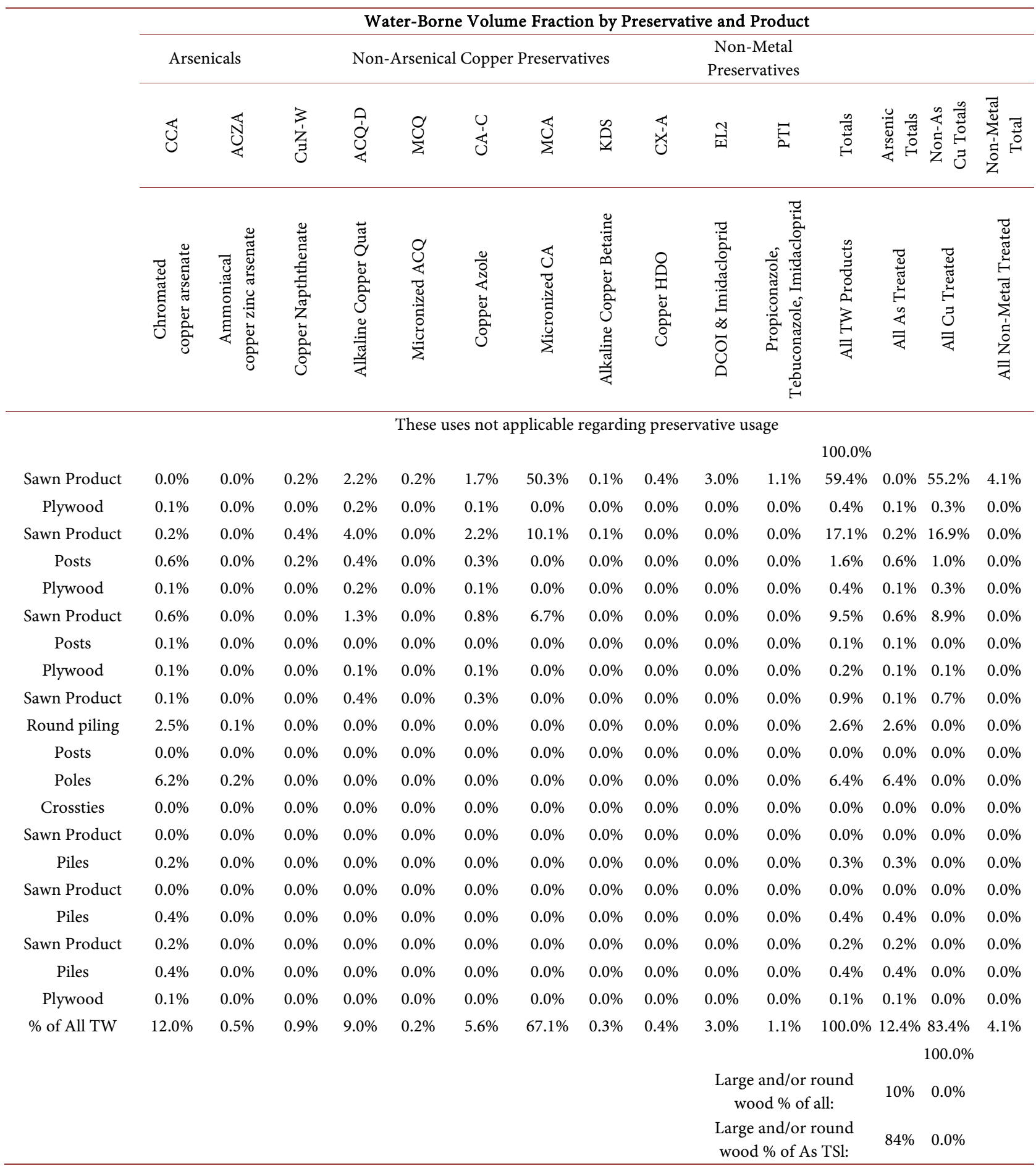


Table 7. Average preservative retentions by commodity.

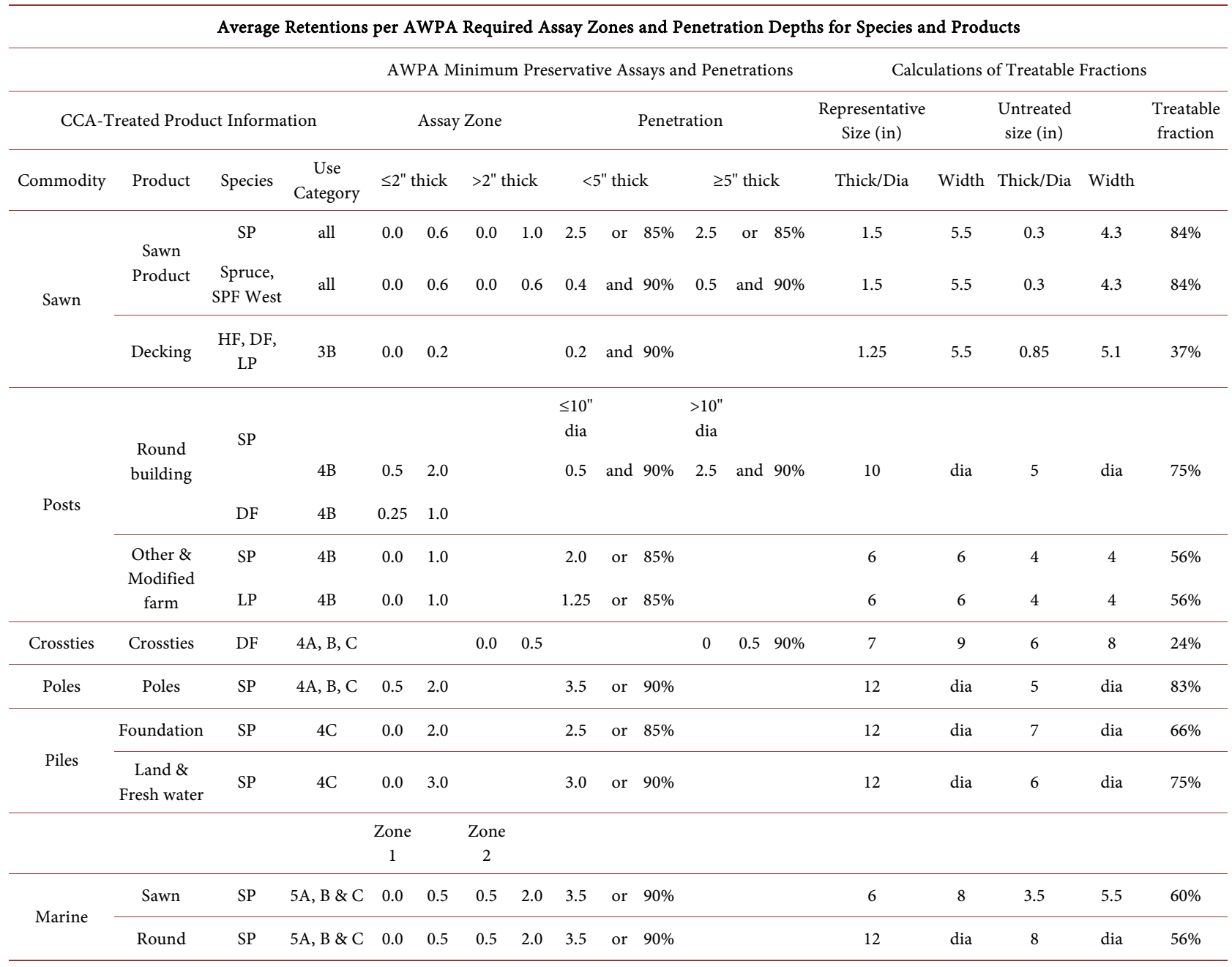

Table 8. Metal fractions in preservatives.

\section{Metal Fractions in Preservatives}

\begin{tabular}{|c|c|c|c|c|}
\hline Preservative and Components & Component Fraction & Metal Atomic Weight & Oxygen Atomic Wt. & Component Fraction \\
\hline \multicolumn{5}{|l|}{ CCA (AWPA P23-10) } \\
\hline Hexavalent $\mathrm{Cr}$ as $\mathrm{CrO}_{3}$ & $47.50 \%$ & 52 & 48 & $24.70 \%$ \\
\hline Copper as $\mathrm{CuO}$ & $18.50 \%$ & 63.5 & 16 & $14.78 \%$ \\
\hline Arsenic as $\mathrm{As}_{2} \mathrm{O}_{5}$ & $34.00 \%$ & 150 & 80 & $22.17 \%$ \\
\hline \multicolumn{5}{|l|}{ ACQ (AWPA P29-11) } \\
\hline Copper as $\mathrm{CuO}$ & $66.70 \%$ & 63.5 & 16 & $53.28 \%$ \\
\hline \multicolumn{5}{|l|}{ ACZA (AWPA P22-10) } \\
\hline Copper as $\mathrm{CuO}$ & $50 \%$ & 63.5 & 16 & $39.94 \%$ \\
\hline Zinc as $\mathrm{ZnO}$ & $25 \%$ & 65.4 & 16 & $20.09 \%$ \\
\hline Arsenic as $\mathrm{As}_{2} \mathrm{O}_{5}$ & $25 \%$ & 150 & 80 & $16.30 \%$ \\
\hline \multicolumn{5}{|l|}{ KDS (AWPA P55-16) } \\
\hline Copper as $\mathrm{CuO}$ & $47.20 \%$ & 63.5 & 16 & $37.70 \%$ \\
\hline
\end{tabular}




\section{Continued}

CX-A (AWPA P33-18)

\begin{tabular}{|c|c|c|c|c|}
\hline Copper as $\mathrm{CuO}$ & $61.50 \%$ & 63.5 & 16 & $49.12 \%$ \\
\hline Boron as $\mathrm{H}_{3} \mathrm{BO}_{3}$ & $24.50 \%$ & & & \\
\hline $\mathrm{HDO}$ as $\mathrm{HDO}$ & $14.00 \%$ & & & \\
\hline
\end{tabular}

CuN-W (AWPA P34-14)

$\begin{array}{lll}\text { Copper as } \mathrm{Cu} & 5.00 \% & \text { Copper in } \mathrm{CuN}\end{array}$

$\begin{array}{ll}\text { Copper naphthenate } & 48.00 \%\end{array}$

Total $\mathrm{Cu}$

$8.36 \%$

Estimates were made of the product mix through 2003 and after 2003, when applications allowed by the new preservative label eliminated most residential and commercial outdoor lumber uses. The product mix data was used to estimate production of the products by year. A summary of the products treated by each preservative type and by all water-borne preservatives is provided in Table 9.

Note that for the purpose of estimating disposal amounts, the product mix shown in the All Water-Borne column in Table 9 is assumed applicable for arsenic-based preservatives for all years prior to 2004, before the label changes became effective. From 2004 forward, the separate mixes are assumed applicable for all years.

The changes in both total treatment volume and product mix through 2003 to 2004 and forward, due to the label changes, were dramatic. Total CCA treatment volume was reduced from nearly 600 million cubic feet per year to about 100 million cubic feet. Lumber, which was mostly used for decks and related outdoor residential and commercial construction, was reduced from about 384 million cubic feet to about 9 million cubic feet. The volume of wood treated with CCA for utility poles was assumed to remain constant, since that use was not affected by the label changes, at approximately 23 million cubic feet. The relative fraction of wood treated with CCA for that product increased from about $4 \%$ to about $25 \%$.

\subsection{Service Lives}

Since the product mix of arsenical-treated wood has changed dramatically since the 2003 label changes, the average service life has also changed. Although industrial products were also being produced, most of the arsenical treated wood prior to 2004 was for relatively short-lived products such as deck lumber. Currently, most arsenical treatment is for larger, longer lived, industrial products such as utility poles, piling, and marine construction.

Estimation of service lives of products is difficult. In the 2005 evaluation [2], data produced by Saxe [10] on service life of decking was used to develop service 
Table 9. Post-2003 preservative uses by commodity.

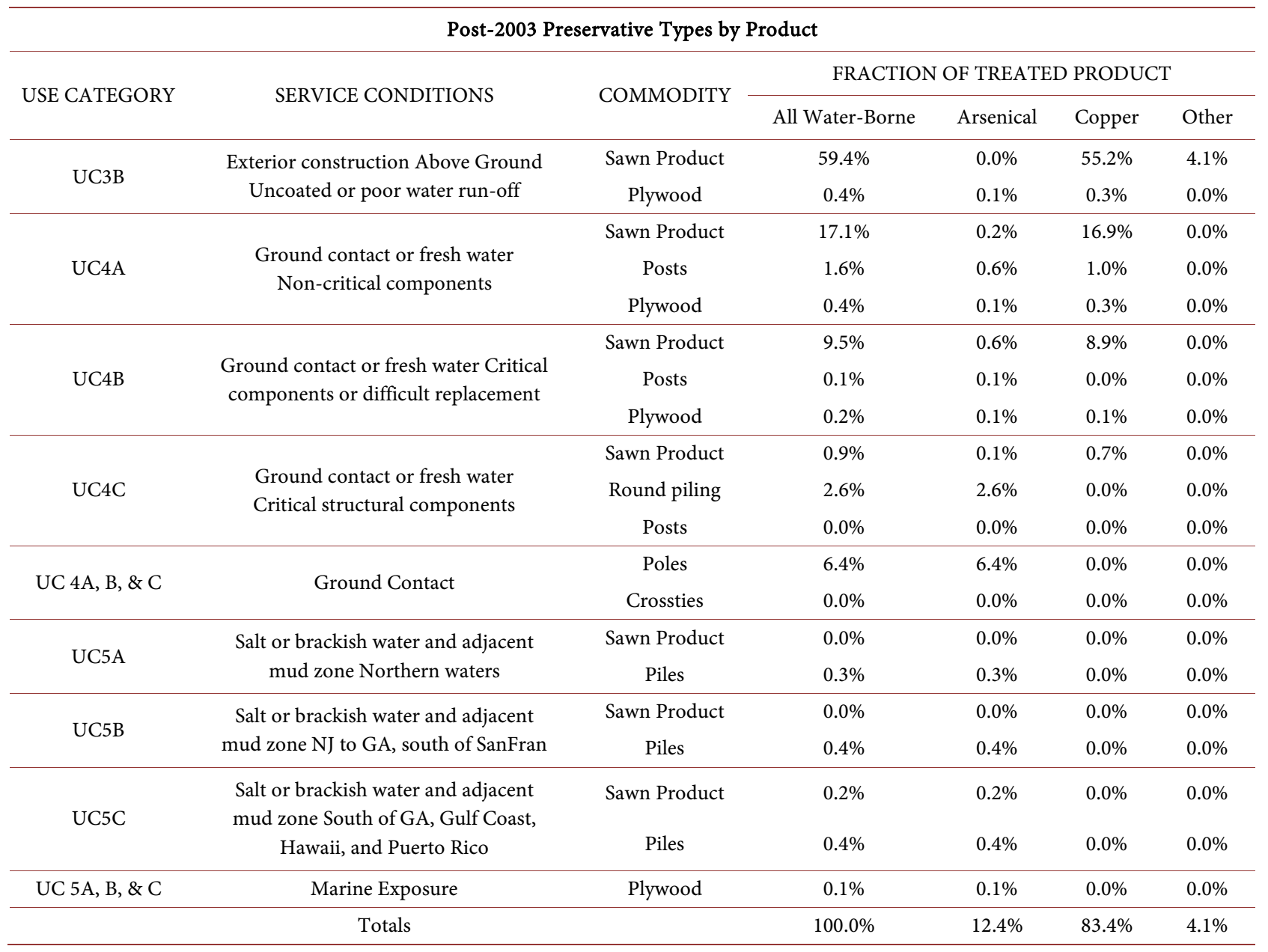

life statistical values for decking, concluding that decks had a 9.45 year average service life with a 5 year standard deviation. It was further concluded that applying a lognormal distribution resulted in the best fit to the Saxe data and logically fit realistic circumstances. For example, the average life of a deck may be about 10 years, but some may last 30 years.

This approach of applying assumed average service lives, standard deviations, and lognormal distributions has been applied to all products treated with water-borne preservatives. These statistical values are summarized in Table 10. Note that longer service lives are estimated for more extreme service conditions because these products typically are thicker and have higher retentions of preservative. This table also includes a column stating the percent of material assumed to be construction waste that would be disposed immediately as part of the construction work.

Fractions of each product reaching end-of-life were calculated using the statistical values shown in Table 8. Results for each category of preservative are shown graphically in Figure 1. Service life associated with each preservative is the result of the product mix for which the preservative is used. This represents 
the current product mix. Note that prior to 2004, the only water-borne treatments were arsenical treatments. In Figure 1, arsenical treatments have longer service lives because they are used only for the heavy-duty industrial type products. Smaller size, less critical or expensive products, such as decking and fencing, are currently treated primarily with the non-arsenical copper and non-metal preservatives that generally have shorter service lives. Prior to 2004, the typical range of service lives for arsenical treated products would match the line for all treated wood, rather than the arsenical line, since all products were then treated with arsenicals. Cumulative fractions of preserved wood products reaching end-of-life are shown numerically by decade in Table 11 .

Table 10. Statistical values for commodities.

\begin{tabular}{|c|c|c|c|c|c|c|c|}
\hline \multirow[b]{2}{*}{ USE CATEGORY } & \multirow[b]{2}{*}{ SERVICE CONDITIONS } & \multirow[b]{2}{*}{ COMMODITY } & \multicolumn{5}{|c|}{ Statistical Values } \\
\hline & & & $\begin{array}{l}\text { Construction } \\
\text { Waste } \\
(\%)\end{array}$ & $\begin{array}{l}\text { Mean } \\
\text { Service } \\
\text { Life } \\
(y r)\end{array}$ & $\begin{array}{l}\text { Standard } \\
\text { Deviation } \\
\quad(\mathrm{yr})\end{array}$ & $\begin{array}{l}\text { Geometric } \\
\text { Mean of } \\
\text { Service Life } \\
\quad(y r)\end{array}$ & $\begin{array}{c}\text { SD of } \\
\text { Lognormal } \\
\text { Service Life } \\
\quad(\mathrm{yr})\end{array}$ \\
\hline $\mathrm{UC1}$ & $\begin{array}{l}\text { Interior construction } \\
\text { Above Ground Dry }\end{array}$ & & \multirow{3}{*}{\multicolumn{5}{|c|}{ These uses not applicable regarding preservative usage }} \\
\hline UC2 & $\begin{array}{l}\text { Interior construction } \\
\text { Above Ground Damp }\end{array}$ & & & & & & \\
\hline UC3A & $\begin{array}{c}\text { Exterior construction Above } \\
\text { Ground Coated \& rapid water runoff }\end{array}$ & & & & & & \\
\hline \multirow{2}{*}{ UC3B } & Exterior construction Above Ground & Sawn Product & $2.5 \%$ & 10 & 5 & 2.2 & 0.47 \\
\hline & Uncoated or poor water run-off & Plywood & $2.5 \%$ & 10 & 5 & 2.2 & 0.47 \\
\hline \multirow{3}{*}{ UC4A } & \multirow{3}{*}{$\begin{array}{c}\text { Ground contact or fresh } \\
\text { water Non-critical components }\end{array}$} & Sawn Product & $2.5 \%$ & 15 & 5 & 2.7 & 0.32 \\
\hline & & Posts & $0.0 \%$ & 20 & 5 & 3.0 & 0.25 \\
\hline & & Plywood & $2.5 \%$ & 15 & 5 & 2.7 & 0.32 \\
\hline \multirow{3}{*}{ UC4B } & \multirow{3}{*}{$\begin{array}{l}\text { Ground contact or fresh water } \\
\text { Critical components } \\
\text { or difficult replacement }\end{array}$} & Sawn Product & $2.5 \%$ & 15 & 5 & 2.7 & 0.32 \\
\hline & & Posts & $0.0 \%$ & 20 & 5 & 3.0 & 0.25 \\
\hline & & Plywood & $2.5 \%$ & 15 & 5 & 2.7 & 0.32 \\
\hline \multirow{3}{*}{ UC4C } & \multirow{3}{*}{$\begin{array}{l}\text { Ground contact or fresh water } \\
\text { Critical structural components }\end{array}$} & Sawn Product & $2.5 \%$ & 30 & 5 & 3.4 & 0.17 \\
\hline & & Round piling & $0.0 \%$ & 50 & 10 & 3.9 & 0.20 \\
\hline & & Posts & $0.0 \%$ & 25 & 5 & 3.2 & 0.20 \\
\hline \multirow{2}{*}{$\mathrm{UC} 4 \mathrm{~A}, \mathrm{~B}, \& \mathrm{C}$} & \multirow{2}{*}{ Ground Contact } & Poles & $0.0 \%$ & 60 & 15 & 4.1 & 0.25 \\
\hline & & Crossties & $0.0 \%$ & 40 & 10 & 3.7 & 0.25 \\
\hline \multirow{2}{*}{ UC5A } & \multirow{2}{*}{$\begin{array}{l}\text { Salt or brackish water and } \\
\text { adjacent mud zone Northern waters }\end{array}$} & Sawn Product & $2.5 \%$ & 40 & 10 & 3.7 & 0.25 \\
\hline & & Piles & $0.0 \%$ & 40 & 10 & 3.7 & 0.25 \\
\hline \multirow{2}{*}{ UC5B } & \multirow{2}{*}{$\begin{array}{l}\text { Salt or brackish water and } \\
\text { adjacent mud zone NJ to GA, } \\
\text { south of SanFran }\end{array}$} & Sawn Product & $2.5 \%$ & 40 & 10 & 3.7 & 0.25 \\
\hline & & Piles & $0.0 \%$ & 40 & 10 & 3.7 & 0.25 \\
\hline \multirow[t]{2}{*}{ UC5C } & $\begin{array}{l}\text { Salt or brackish water and adjacent } \\
\text { mud zone South of GA, Gulf Coast, }\end{array}$ & Sawn Product & $2.5 \%$ & 40 & 10 & 3.7 & 0.25 \\
\hline & Hawaii, and Puerto Rico & Piles & $0.0 \%$ & 40 & 10 & 3.7 & 0.25 \\
\hline UC 5A, B, \& C & Marine Exposure & Plywood & $2.5 \%$ & 40 & 10 & 3.7 & 0.25 \\
\hline
\end{tabular}


Table 11. Cumulative end-of-life by preservative class.

\begin{tabular}{cccccccccccc}
\hline $\begin{array}{c}\text { Cumulative Fraction of Treated } \\
\text { Wood Reaching End-of-Life by Decade }\end{array}$ & \multicolumn{10}{c}{ End-of-Life at Years After Treatment } \\
\cline { 2 - 11 } & 0 & 10 & 20 & 30 & 40 & 50 & 60 & 70 & 80 & 90 & 100 \\
\hline All Treated Wood & $2.2 \%$ & $40.4 \%$ & $81.4 \%$ & $88.7 \%$ & $91.0 \%$ & $93.9 \%$ & $96.6 \%$ & $98.4 \%$ & $99.3 \%$ & $99.7 \%$ & $99.9 \%$ \\
Arsenical Treated Wood & $0.3 \%$ & $1.7 \%$ & $10.9 \%$ & $17.0 \%$ & $28.7 \%$ & $51.1 \%$ & $73.5 \%$ & $88.0 \%$ & $95.4 \%$ & $98.7 \%$ & $100.0 \%$ \\
Non-Arsenic Copper Treated Wood & $2.5 \%$ & $45.2 \%$ & $91.3 \%$ & $98.9 \%$ & $99.9 \%$ & $100.0 \%$ & $100.0 \%$ & $100.0 \%$ & $100.0 \%$ & $100.0 \%$ & $100.0 \%$ \\
Non-Metal Treated Wood & $2.5 \%$ & $60.4 \%$ & $95.7 \%$ & $99.5 \%$ & $99.9 \%$ & $100.0 \%$ & $100.0 \%$ & $100.0 \%$ & $100.0 \%$ & $100.0 \%$ & $100.0 \%$ \\
\hline
\end{tabular}

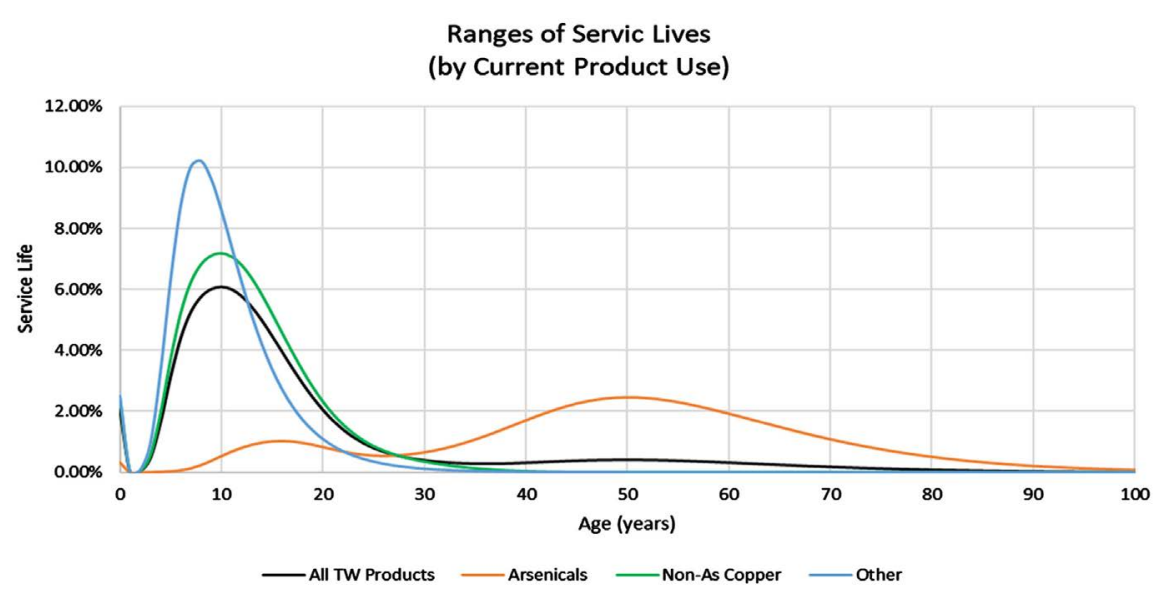

Figure 1. Service lives of products.

Table 11 indicates that about $90 \%$ of all water-borne preserved wood products reach end-of-life by age 40 . However, $90 \%$ of the copper and other preserved products have reached their end-of-life by only age 20 .

\subsection{Disposal}

Annual disposal of water-borne preservative treated wood is calculated using annual treatment times the fraction disposed by age following treatment. This was accomplished by creating a spreadsheet with years of disposal from 1950 to 2050 horizontally and years of treatment from 1950 to 2050 vertically. For each treatment times disposal cell, disposal volume was calculated using the fraction disposed for all treated wood. Columns were summed for each disposal year to calculate the total amount of disposal in that year. Obviously, no disposal could occur for years prior to the product's treatment year.

A nearly identical spreadsheet was created for arsenical-treated wood. It differed for treatment years from 2004 through 2050 in that the fraction of disposal by age reflected to current product mix for arsenical-treated wood rather than for all treated wood. Annual treatment and disposal volumes of all and arsenical treated wood products are listed for each year in Table 1 and are shown graphically in Figure 2. (These spreadsheets are too large to include, so are available from the author.) Note that a similar graph showing the impact of the label change of 2003 on CCA-treated wood disposal was created in 2007 [10]. 




Figure 2. Annual treatment and disposal.

In Figure 2, prior to 2004, treatment and disposal of all water borne treated wood is the same as for arsenical treated wood. In 2004, the lines diverge as treatment with arsenicals declines precipitously from approximately 600 million to about 100 million cubic feet per year. Disposal of arsenical preserved products declines more gradually as previously treated products reach end-of-life and as ongoing treatments occur with a different, longer-lived product mix. However, the decline in arsenical treated products from approximately 400 million cubic feet in 2008 to approximately 100 million cubic feet in 2020 is still dramatic.

The types of arsenical treated products reaching the end-of-life stage from now forward is important to understand as it relates to solid waste disposal and potential recycling of biomass to mulch or other products. It has been 17 years since the label changes restricted arsenical treatments to industrial type products. As of 2020, 59\% of all preserved wood products and $76 \%$ of arsenical treated products have reached their end-of-life and been disposed. Of the arsenical treated products being disposed from 2020 forward, approximately $84 \%$ consist of poles, piles, and other heavy-duty products (posts, piles, poles, and marine timbers) that are round and/or of large dimensions relative to residential and commercial lumber. Thus, such arsenical treated products being disposed can easily be visually distinguished and can be kept separate or be manually separated from other wood wastes. Most could be managed separately by the generators, so that most would never enter the general waste stream.

Based on the service life statistics, within 20 years of treatment approximately $95 \%$ of above-ground and $85 \%$ of ground-contact treated lumber (Use Categories $3 \mathrm{~B}$ through $4 \mathrm{~B}$ ) for residential and commercial uses, such as for decks and fencing, has reached its expected end-of-life and been disposed. Thus, only a very small fraction of this arsenical treated lumber produced prior to 2004 remains in service. 


\subsection{Uncertainty}

Due to limited company involvement, the survey results are incomplete, especially for the non-metal type preservatives. Results of the preservative suppliers' survey indicate only approximately $2 \%$ of total treated wood by non-metal preservatives. In contrast, Solo-Gabriele reported [9] that of all treated wood, approximately $11 \%$ was by EL2 and $2 \%$ by PTI. The survey results seem low and the Solo-Gabriele estimates seem high. For this report, the author estimated the fractions of preservatives reported so that total U.S. preservatives use could be estimated. For the non-metal preservatives, $2 \%$ are estimated to be included in the survey results. Where higher reporting is estimated, reported non-arsenical copper preservatives are estimated to be $80 \%$ of totals and arsenical preservatives reported amounts are estimated to be $95 \%$ of totals. With these adjustments, non-metal preservative treated wood represents approximately $4 \%$ in 2016 and 6\% in 2018 of all treated wood compared to approximately 13\% estimated by Solo-Gabriele. The results in this report, as shown in Table 2, seem reasonable.

Annual treatment volumes, as shown in Table 1, required judgement to estimate amounts for years and preservative types where no relevant data was available. In particular, for the years from 2002 through 2015, it was necessary to simply enter estimates that would represent a reasonable total treatment volume and annual trend. Non-arsenical copper treatments are assumed to begin in 2002, ramp up rapidly with the label change restricting use of CCA through 2005, and then remain consistent through 2015. Non-metal preservatives are assumed to begin use in 2004 and ramp up gradually through 2015. Values for 2015 are chosen to be reasonably close to the estimated 2016 values resulting from this survey.

The product mix of non-arsenical copper preservatives from 2004 through 2015 has evolved from dissolved copper preservatives, such as ACQ, to micronized copper, as in MCA. This evolution is not included. Rather, the current mix is assumed constant. This assumption likely introduces some small inaccuracy related to the amounts of copper in waste.

Future treatment volumes are simply assumed to increase at $1 \%$ annually, beginning with the volumes estimated for 2018 for each preservative type. Future changes are likely, but unknowable.

Similarly, technology evolution related to recycling wastes to energy and other options are likely to present new options for waste management other than landfill disposal. Impacts of such technologies remain unknowable.

As a result of the above and other sources of uncertainty, annual treatment volumes and disposal volumes should be considered rough estimates, generally with an accuracy of about plus or minus $25 \%$.

\section{Conclusions}

Water-borne preservative treated wood has been and is likely to continue to be widely used. However, due to the label changes made in 2003 for arsenical wood 
preservatives, the mix of products now treated with water-borne preservatives has changed dramatically. Prior to 2004, nearly all water-borne preservative was arsenic-based. From 2004 forward, less than 20\% (by volume of treated wood) is treated with arsenic-based preservative. 2018 production of water-borne preserved wood products is estimated, based on the survey of preservative suppliers, to be 70 million cubic feet of arsenical treatments, 532 million cubic feet of non-arsenic copper treatments, and 42 million cubic feet of non-metal treatments for a total of 644 million cubic feet.

As water-borne preserved wood products reach the end of their service lives, they are assumed to be disposed. As more preserved wood enters use, more also enters the disposal sector. However, as is clear from Figure 2, most of the arsenic-based volume treated prior to 2003 has already been disposed. As of 2019, over $75 \%$ of all arsenic treated wood has been disposed. National disposal of arsenic treated wood peaked in 2007-2008 at approximately 426 million cubic feet per year, is at approximately 110 million cubic feet now, and will reach a minimum of approximately 42 million cubic feet per year in 2030. As noted above, nearly all arsenical preserved wood being disposed will be large, heavy duty poles, piles, and timbers that can easily be kept separate or be separated from other wastes.

\section{Acknowledgements}

This work has been supported and funded by the Treated Wood Council (TWC). The TWC provides government support to all segments of the treated wood industry.

\section{Conflicts of Interest}

The author declares no conflicts of interest regarding the publication of this paper.

\section{References}

[1] AWPI (1997) The 1996 Wood Preserving Industry Statistical Report. American Wood Preservers Institute.

[2] Smith, S.T. (2005) CCA Treated Wood Production, Uses, and Disposal. Wood Preservatives Science Council, Not Published.

[3] (2016) Production Volumes and Benefits of CCA-Treated Wood. American Chemistry Council, Biocides Panel, Arsenical Wood Preservers Task Force, Not Published.

[4] Jones, A.S., Marini, J., Solo-Gabriele, H.M., Robey, N.M. and Townsend, T.G. (2019) Arsenic, Copper, and Chromium from Treated Wood Products in the U.S. Disposal Sector. Waste Management, 87, 731-740. https://doi.org/10.1016/j.wasman.2019.03.004

[5] AWPI (1996) The 1995 Wood Preserving Industry Statistical Report. American Wood Preservers Institute.

[6] Micklewright, J.T. (1998) Wood Preservation Statistics 1997. American Wood Preservers' Association. 
[7] Smith, S.T. (2011) Economics of Treated Wood in Aquatic Environments. In: Morrell, J.J., Brooks, K.M., C.M., Davis, Eds., Managing Treated Wood in Aquatic Environments, Forest Products Society.

[8] American Wood Protection Association (2018) Book of Standards. AWPA, Birmingham, AL.

[9] Solo-Gabriele, H., Jones, A., Marini, J., Townsend, T. and Robey, N. (2016) Trends in Waterborne Treated Wood Production and Implications for Wood Waste Disposal. American Wood Protection Association, San Juan, Puerto Rico.

[10] Saxe, J.K., Wannamaker, E.J., Conklin, S.W., Shupe, T.F. and Beck, B.D. (2007) Evaluating Landfill Disposal of Chromated Copper Arsenate (CCA) Treated Wood and Potential Effects on Groundwater: Evidence from Florida. Chemosphere, 66, 496-504. https://doi.org/10.1016/j.chemosphere.2006.05.063 